\title{
20 Jahre FMH-Gutachterstellen - Jahresbericht für die Berichtsperiode 2001
}

Fürsprecher Hanspeter Kuhn, stv. Generalsekretär

1 Der Jahresbericht für 2000 erschien in der männlichen Form. Dieses Jahr sind turnusgemäss die Frauen an der Reihe. Das andere Geschlecht ist jeweils mitgemeint.

2 Eröffnung der Vernehmlassung: FMH-Gutachterstelle. Berichtsperiode 2000. Schweiz Ärztezeitung 2001;82(29/30):1585-94; Publikation des neuen Reglements: Zur Revision Reglement FMHGutachterstelle Schweiz Ärztezeitung 2002;83(4):125-33.
Seit 1982 wurden insgesamt 2587 FMH-Gutachten zur Frage eines Behandlungs- oder Diagnosefehlers erstellt. Zum ersten Mal seit Bestehen der Gutachterstelle haben die Gutachterinnen [1] in über 50\% der Fälle einer der beiden Gutachterstellen einen Behandlungsfehler festgestellt, nämlich in 37 von 67 Fällen (Gutachterstelle Bern für die Deutsche Schweiz und das Tessin).

Die FMH-Gutachterstelle ist nicht für alle Streitigkeiten zuständig. Ihre Aufgabe ist es, dann ein Gutachten in Auftrag zu geben, wenn die Patientin vermutet, der Ärztin oder dem Spital sei ein Diagnose- oder Behandlungsfehler unterlaufen, der zu einem Gesundheitsschaden geführt hat, und wenn zudem zwischen Patientin und Haftpflichtversicherer von Ärztin oder Spital keine Einigung ohne Gutachten möglich war.

Es fällt auf, dass die Zahl der Fälle, in denen die Gutachterinnen einen Fehler feststellen, zwischen den beiden Gutachterstellen in Bern und in Lausanne deutlich unterschiedlich sind. Dies führt zur Frage, ob

- in der deutschen Schweiz und im Tessin die Haftpflichtversicherer zu oft zur Annahme neigen, es liege kein Fehler vor (und die Gutachterin dann einen Fehler feststellt), und

- ob umgekehrt in der Romandie die Patientinnen und/oder ihre Anwältinnen zu rasch vermuten, es liege ein Fehler vor (und die Gutachterin dann keinen Fehler feststellt).

Nach aussen stand die Berichtsperiode im Zeichen der Reglementrevision [2]. Intern war das Jahr gekennzeichnet vom Hinterfragen aller Abläufe im Hinblick auf die Optimierung von Qualität und Zeitdauer der Gutachtensvorbereitung und -durchführung.

\section{Statistik der Gutachterstelle für das Berichtsjahr 2001}

\section{Methode}

Seit Bestehen der Gutachterstelle geben die Gutachterinnen bei Abschluss ihres Gutachtens der Gutachterstelle an, ob ein Diagnose- oder Behandlungsfehler im Gutachten bejaht oder verneint wird.

Die Zuordnung dieser Angabe zum entsprechenden Fachgebiet erfolgt danach durch die Leiterin der Gutachterstelle. Bei multidisziplinären Gutachten erfolgt die Zuordnung zum soweit erkennbar am intensivsten betroffenen Fachgebiet. Beispiel: Ist ein Gutachterinnenteam primär für die Orthopädie und sekundär für die Anästhesie eingesetzt worden, und wird ein Fehler in der Orthopädie bejaht und in der Anästhesie verneint, so wird das Gutachten der Kategorie «Orthopädie, Fehler bejaht» zugeordnet, nicht aber «Anästhesie, Fehler verneint». Die Statistik spiegelt damit primär das Resultat für die Patientin, hingegen nicht vollumfänglich das Mass der geleisteten Untersuchungsarbeit der Gutachterinnen.

Nicht statistisch erfasst werden die Schlussfolgerungen der Gutachterinnen zu Schaden und Kausalität zwischen Fehler und Schaden. Eine statistische Auswertung dieser Frage nach dem einfachen Muster «ja» oder «nein» würde einen falschen Eindruck erwecken, weil die Auswirkungen eines festgestellten Fehlers quantitativ höchst unterschiedlich ausfallen können. Sie reichen von «keine feststellbare nachteilige Folge» bis zu «hat den Tod der Patientin verursacht». 


\section{Resultate}

Tabelle 1

Übersicht globale Zahlen 1982-2001.

\begin{tabular}{|c|c|c|c|c|}
\hline & Erstellte Gutachten & $\begin{array}{l}\text { Behandlungs-/ } \\
\text { Diagnosefehler } \\
\text { bejaht }\end{array}$ & $\begin{array}{l}\text { Behandlungs-/ } \\
\text { Diagnosefehler } \\
\text { verneint }\end{array}$ & $\begin{array}{l}\text { Behandlungs-/ } \\
\text { Diagnosefehler } \\
\text { unbestimmt }\end{array}$ \\
\hline Lausanne und Bern 1982-2000 & 2451 & 720 & 1650 & 81 \\
\hline Büro Bern 2001 & 67 & 37 & 30 & - \\
\hline Büro Lausanne 2001 & 69 & 26 & 43 & - \\
\hline Total 1982-2001 & $2587(100 \%)$ & $783(30,3 \%)$ & $1723(66,6 \%)$ & $81(3,1 \%)$ \\
\hline
\end{tabular}

Tabelle 2

Ergebnisse nach Fachgebieten 1982-2001.

\begin{tabular}{|c|c|c|c|c|}
\hline Fachgebiet & Erstellte Gutachten & $\begin{array}{l}\text { Behandlungs-/ } \\
\text { Diagnosefehler } \\
\text { bejaht }\end{array}$ & $\begin{array}{l}\text { Behandlungs-/ } \\
\text { Diagnosefehler } \\
\text { verneint }\end{array}$ & $\begin{array}{l}\text { Behandlungs-/ } \\
\text { Diagnosefehler } \\
\text { unbestimmt }\end{array}$ \\
\hline Allgemeinchirurgie & 680 & 227 & 429 & 24 \\
\hline Allgemeinmedizin & 189 & 63 & 118 & 8 \\
\hline Anästhesiologie & 95 & 26 & 67 & 2 \\
\hline Dermatologie & 24 & 8 & 14 & 2 \\
\hline Gastroenterologie & 9 & 1 & 8 & - \\
\hline Gynäkologie & 311 & 109 & 196 & 6 \\
\hline Handchirurgie & 37 & 11 & 25 & 1 \\
\hline Herz- + thorakale Gefässchirurgie & 16 & 2 & 13 & 1 \\
\hline Innere Medizin & 167 & 40 & 123 & 4 \\
\hline Kardiologie & 9 & 4 & 5 & - \\
\hline Kieferchirurgie & 17 & 3 & 14 & - \\
\hline Kinderchirurgie & 13 & 4 & 9 & - \\
\hline Kinderpsychiatrie & 1 & - & 1 & - \\
\hline Nephrologie & 2 & - & 2 & - \\
\hline Neurochirurgie & 55 & 16 & 37 & 2 \\
\hline Neurologie & 18 & 5 & 12 & 1 \\
\hline Notfallchirurgie & 2 & 1 & 1 & - \\
\hline Onkologie & 5 & 3 & 2 & - \\
\hline Ophthalmologie & 100 & 25 & 71 & 4 \\
\hline Oto-Rhino-Laryngologie ORL & 96 & 19 & 74 & 3 \\
\hline Orthopädische Chirurgie & 444 & 139 & 294 & 11 \\
\hline Pädiatrie & 47 & 18 & 26 & 3 \\
\hline Pathologie & 3 & 2 & 1 & - \\
\hline Physikalische Medizin + Rehabilitation & 13 & 3 & 9 & 1 \\
\hline Plastische + Wiederherstellungschirurgie & 117 & 27 & 88 & 2 \\
\hline Pneumologie & 1 & 1 & - & - \\
\hline Psychiatrie & 10 & 3 & 7 & - \\
\hline Radiologie & 34 & 11 & 20 & 3 \\
\hline Rheumatologie & 8 & 2 & 6 & - \\
\hline Urologie & 60 & 9 & 48 & 3 \\
\hline Viszeralchirurgie & 4 & 1 & 3 & - \\
\hline Total & 2587 & 783 & 1723 & 81 \\
\hline
\end{tabular}


Längsschnitt: Im Zwanzigjahresverlauf wurde in 30\% der Fälle ein Fehler festgestellt, in 67\% verneint, und in $3 \%$ blieb die Fehlerfrage letztlich offen.

Historischer Querschnittsvergleich: Im August 1983 konnte erstmals über die von März 1982 bis April 1983 an die Hand genommenen 84 Fälle berichtet werden, von denen 37 abgeschlossen waren. In sechs der 37 Fälle wurde ein Fehler bejaht, in zwei Fällen musste die Frage offen bleiben, und in 29 wurde ein Fehler klar verneint [3]. Ende 1991, also nach zehn Jahren FMH-Gutachterstelle, stand der Zähler auf total 905 Gutachten. In 212 Fällen war bis 1991 ein Fehler bejaht worden, in 642 verneint, 51 Fälle blieben letztlich offen [4].

\section{Gutachten über mehr als einen Fachbereich}

Die moderne Medizin ist komplex geworden. Das historische Bild der Ärztin, die als Einzelkämpferin die Patientin behandelt, prägt auch die Tätigkeit der Gutachterstelle nicht mehr. In rund der Hälfte aller Fälle des letzten Jahres ging es um die Analyse von Spitalbehandlungen, und in vielen anderen Fällen war eine Behandlungskette von mehreren praktizierenden Ärztinnen zu untersuchen. Konkret waren für die im 2001 erstatteten Gutachten die folgenden Begutachtungsteams im Einsatz:

- Allgemeinmedizin - Radiologie (Gutachterstelle Lausanne $=$ nachfolgend $\mathrm{L}$ );

- Allgemeinmedizin - Radiologie - Pädiatrie (Gutachterstelle Bern = nachfolgend $\mathrm{B})$;

- Allgemeinmedizin - Kieferchirurgie (B);

- Anästhesiologie - Innere Medizin (L);

- Anästhesiologie - Gynäkologie (B);

- Chirurgie - Gastroenterologie - Allgemeinmedizin - Innere Medizin (L);

- Chirurgie - Urologie - Radiologie (L);

- Chirurgie - Gynäkologie (B, 2x);

- Chirurgie - Radiologie (B);

- Gynäkologie - Radiologie (B);

- Gynäkologie - Pathologie - Allgemeinmedizin (B);

- Herzchirurgie - Kardiologie - Transfusionsmedizin (L);

- Neurochirurgie - Innere Medizin (L);

- Innere Medizin - Radiologie (B);

- ORL - Dermatologie (L);

- Orthopädie-Anästhesie (L, B); zusätzlich mit Neurologie (B);

Gutachterstelle für Ärztehaftpflichtfälle. Schweiz Ärztezeitung 1983;64(31):1159-60.

4 Kuhn HP. Aussergerichtliche Gutachterstelle der FMH. 10. Jahresbericht für 1991 Schweiz Ärztezeitung 1992; 73(20):766-7.

\section{Diskussion}

Begrenzte Aussagekraft der Gutachterstellenfälle Die Statistik der Gutachterstelle ist nur begrenzt repräsentativ für die Spital- und Arzthaftpflichtsituation in der Schweiz. Die letztes Jahr im Rahmen der beiden FMH-Gutachterstellen erstatteten 136 Gutachten sind beispielsweise in Relation zu setzen zu den rund 30 bis 40 Haftpflichtfällen, die typischerweise jährlich in einem einzelnen grossen nicht universitären Kantonsspital anfallen.

Die Gutachterstelle kann nur über ihre eigenen Fälle berichten. Wir wissen nicht, wie viele Fälle von den Versicherern direkt ohne Gutachten erledigt werden, wie viele mit einem Gutachten ausserhalb der FMH-Gutachterstelle, und wie viele vor Gericht.

«Bern»: 37 Fälle mit bejahtem Fehler, 30 mit verneintem Fehler - es kommt auf den Case-Mix an Die absoluten Fallzahlen sind bei der Berner Gutachterstelle seit 1996 kontinuierlich zurückgegangen. Im Gegenzug ist die Fehleranerkennungsrate gestiegen: im Jahr 2000 erstmals auf 50\%, und im Berichtsjahr 2001 ist diese Zahl von den Gutachterinnen anerkannter Fehler nun klar überschritten worden. Wer mit der Arbeit der FMH-Gutachterstelle vertraut ist, nimmt dieses Ergebnis ohne Erstaunen zur Kenntnis. Seit Jahren haben wir darauf hingewiesen, dass die Zahlen der Jahresstatistik vor allem davon abhängen, wie die Fälle gelagert sind, in denen ein Gutachten in Auftrag gegeben wird.

Zur Rolle der Patientenanwältinnen und -beraterinnen: Die Fehleranerkennungsquote ist nach dem, was wir über die Arbeitsweise der Patientenanwältinnen erfahren, eindeutig davon abhängig, wie gut deren medizinisches Beratungsnetz ist. Im Bereich der Medizinhaftpflicht ist die Anwältin primär Übersetzerin medizinischen Wissens (nicht anders, als beispielsweise eine Patentanwältin sich wesentlich um technische Fragen kümmern und dafür ein Beratungsnetz aufbauen muss). Es ist unverzichtbar, vor grossen juristischen Aufwendungen auf Patientinnenseite eine medizinische Lagebeurteilung $\mathrm{zu}$ veranlassen. Das heisst konkret, aufgrund der Krankengeschichte und der Röntgenbilder zwischen Patientin oder Anwältin und beratenden Ärztinnen zu überlegen, ob es richtig ist, dass die Patientin an ihrer ersten Vermutung festhält, es sei wohl ein Fehler in der Behandlung unterlaufen.

Von dieser (selbst)kritischen internen medizinischen Analyse auf Patientinnenseite hängt 
bereits ab, wie frau versucht, mit dem Haftpflichtversicherer von Spital oder Ärztin eine direkte Einigung ohne Gutachten zu erreichen. Und auch dann, wenn der Haftpflichtversicherer sich nicht auf eine Einigung ohne Gutachten einlässt, ist die medizinische Lagebeurteilung auf Patientinnenseite entscheidend dafür, ob und wie ein Gutachten organisiert werden soll, und zwar unabhängig davon, ob man für die Gutachtensphase die FMH-Gutachterstelle einschaltet, oder direkt zwischen den Parteien eine Gutachterin oder ein -team sucht und beauftragt. Den Patientinnen ist deshalb zu empfehlen, ihre Anwältin danach auszuwählen, ob und mit welchen medizinischen Beraterinnen sie zusammenarbeitet.

Die eigentlich rechtlichen Fragen, für die die Anwältinnen selbst die nötige Fachkompetenz haben, stellen sich erst, nachdem die medizinische Frage nach Untersuchungs- oder Behandlungsfehler und dessen medizinischen Folgen für den Gesundheitszustand der Patientin geklärt ist.

Zur Rolle der Haftpflichtversicherer, der Spitalträger, der Sozialversicherer und des Bundesrats: Die unseres Erachtens hohe Fehleranerkennungsquote der Fälle der Gutachterstelle in Bern führt zur Frage, ob einige Haftpflichtversicherer unnötigerweise auf einem FMH-Gutachten beharrten in Fällen, die bei vernünftiger Lagebeurteilung direkt hätten erledigt werden können und sollen.

Ohne detaillierte Analyse der Fälle, vorzugsweise im Rahmen von «closed-claims studies» der medizinischen Fachgesellschaften, lässt sich diese Frage nur stellen, aber nicht beantworten.

Wichtig und naheliegend ist immerhin ein Hinweis: Wenn die Spitalträger - also die Kantone und Gemeinden -, die Sozialversicherer und der Bundesrat (letzterer als oberste Instanz in Tarifstreitigkeiten) die verfügbaren Ressourcen der Spitäler weiterhin wie die sprichwörtliche Zitrone auspressen, ist voraussehbar, dass sich der Druck auf das Spital erhöht, die Haftpflichtversicherung mit dem günstigsten Angebot zu wählen. Dies birgt das offensichtliche Risiko in sich, dass der zum Zuge gekommene Versicherer sehr hart abwägen muss, welche Patientinnen den Willen und die Ressourcen haben, einer vermuteten Fehlerfrage auf den Grund zu gehen: Über die Phase der Verhandlungen noch ohne Gutachten, die Einholung eines Gutachtens bis schlimmstenfalls zur Klageeinreichung vor Gericht. Diese Entwicklung ist weder den Patientinnen noch den Spitalärztinnen und dem Pflegepersonal zu wünschen. Wir wiederholen, was wir bereits im letztjährigen Jahresbericht
Die Patientin vermutet einen Behandlungs- oder Diagnosefehler was soll die betroffene Ärztin tun?

1. Mit der Patientin in Ruhe einen für beide Seiten passenden Besprechungstermin vereinbaren. Will sie eine Person ihres Vertrauens zu dieser Besprechung mitnehmen?

2. Den Fall rasch (!) der Haftpflichtversicherung melden und das weitere Vorgehen besprechen; vorher das Einverständnis der Patientin dafür einholen (mündlich, Eintrag in Krankengeschichte). Die Benachrichtigung des Haftpflichtversicherers bedeutet nicht das Eingeständnis eines Fehlers.

3. Im öffentlichen Spital: Mit der Vorgesetzten und mit der zuständigen Person in der Spitaldirektion rasch eine interne Standortbestimmung vornehmen.

4. Möglichst bald aus dem Gedächtnis ein möglichst vollständiges Protokoll der entscheidenden Untersuchungs- oder Behandlungsphasen diktieren oder aufschreiben («den Film noch einmal ablaufen lassen»).

5. Der Patientin auf Verlangen ohne weiteres und unentgeltlich eine Fotokopie der Krankengeschichte zur Verfügung stellen. Röntgenbilder gegen Quittung der Patientin ausleihen.

6. Falls das Gespräch zwischen Ärztin und Patientin keine Klarheit brachte: Der Patientin aufzeigen, mit wem sie den Fall weiter besprechen kann, um sich eine eigene Meinung bilden zu können.

7. Die Patientin nicht an die Gutachterstelle verweisen, ohne vorher (ohne Bekanntgabe der Patientenidentifikation) den Fall telefonisch mit der Leiterin der Gutachterstelle zu besprechen.

8. Braucht die betroffene Ärztin selbst eine Anwältin? Der Haftpflichtversicherer ist nicht nur dafür da, zu bezahlen, wenn ein Haftpflichtfall vorliegt, sondern auch dafür, ungerechtfertigte Haftpflichtansprüche abzuwehren. Insoweit braucht die Ärztin grundsätzlich keine eigene Anwältin. Hingegen zeigt die Erfahrung, dass es je nach Fall sinnvoll sein kann, die Situation mit einer aussenstehenden Anwältin unverbindlich zu besprechen. Eine solche Standortbestimmung kann unter anderem Ängste und Verunsicherungen abbauen. In der Regel übernimmt aber der Haftpflichtversicherer diese Kosten nicht. 
ausgeführt haben: Notwendig sind nicht zusätzliche Beweiserleichterungen für den Patienten, sondern schlicht und einfach genügend finanzielle Rückstellungen beim Haftpflichtversicherer, die allein ihm eine rasche und faire Schadenserledigung erlauben.

Auffallend für die Gutachterstelle Bern sind zwei weitere Aspekte:

- Fälle, in denen von Anfang an klar war, dass ein multidisziplinäres Gutachterinnenteam eingesetzt werden muss, führten überproportional oft zur gutachterlichen Feststellung eines Fehlers, konkret in 13 der 15 multidisziplinären Fälle.

- Fälle, bei denen die Vorbereitungen aufgrund unvollständiger und/oder diffuser, unklarer Anträge mühsam und ausgesprochen aufwendig waren, führten fast nie zur Feststellung eines Fehlers.

Diese Information kann für die betroffenen Patientinnen, Anwältinnen und Haftpflichtversicherer bereits im Vorfeld einer Begutachtung hilfreich sein.

\section{«Lausanne»: hohe Inzidenz an Gutachten; in zwei Drittel der Fälle keinen Fehler festgestellt} Für die Romandie fällt zweierlei auf: Erstens wurden auch letztes Jahr im Landesvergleich überproportional viele Gutachten erstellt, nämlich mehr als für die ganze übrige Schweiz. Zweitens bleibt die Rate der festgestellten Fehler seit Jahren auf rund einem Drittel der Fälle stehen. Das bedeutet: Die Patientinnen, ihre Anwältinnen und gegebenenfalls ihre medizinischen Beraterinnen täuschen sich in der Lagebeurteilung vor Erstattung des Gutachtens doppelt so häufig wie die Haftpflichtversicherer.

Damit steht unseres Erachtens die Frage im Raum, ob die Patientinnen (bzw. ihre Anwältinnen) zuweilen die medizinische Situation gar nicht oder zu wenig gründlich abklären, bevor sie einen Antrag bei der Gutachterstelle einreichen. Es ist wohl wahr, dass die Bearbeitungsgebühr für das eigentliche Gutachten aus sozialen und politischen Gründen tief angesetzt ist und die tatsächlichen Kosten der Fallvorbereitung durch die Gutachterstelle und der Arbeit der Gutachterin bei weitem nicht deckt. Aber es ist $\mathrm{zu}$ bedenken, dass die meisten Patientinnen ihren Antrag durch eine praktizierende Anwältin schreiben lassen. Oft beträgt deren Honorar bis und mit Gutachtensphase bereits mehrere tausend Franken. Angesichts der beachtlichen finanziellen und menschlichen Ressourcen, die ein Gutachten bei allen Beteiligten (Patientin, Anwältin, Versicherer, Gutachterstelle, Delegier- ter der Fachgesellschaft und insbesondere Gutachterin) verbraucht, wäre ernsthaft zu prüfen, ob hier nicht konkrete Änderungen möglich wären: - Die Patientinnen bzw. Anwältinnen sollten als ersten Schritt bessere medizinische Vorabklärungen organisieren, bevor Anwaltskosten und Gutachtensaufwendungen im grossen Stil generiert werden.

- Die kantonalen Ärztegesellschaften könnten zudem überlegen, ob nicht denkbar wäre, pro Kanton oder gemeinsam im Rahmen der Romandie ein Netz von Second-opinion-Ärztinnen aufzubauen, dass dem Ombudsmann der Ärztegesellschaft, staatlichen Ombudspersonen und Beschwerdekommissionen, Patientinnen- und Versichertenorganisationen und den Patientinnenanwältinnen als Ansprechpartner zur Verfügung gestellt werden könnte. Aufgabe dieser Ärztinnen wäre nicht, ein Gutachten zu erstellen, sondern sich in Kenntnis der von der Patientin eingeholten Krankengeschichte dazu zu äussern, ob wohl die Erfolgserwartungen der Patientin zu hoch waren, oder ob und was bei der Untersuchung oder Behandlung nicht rund gelaufen sein könnte. Der wichtige Beitrag der Ärztegesellschaft bestünde dabei nicht in der Finanzierung dieser Tätigkeit, sondern in der Bezeichnung von Ärztinnen, die in der Lage und bereit sind, eine solche Aufgabe auszuüben - und damit früh und kosteneffizient einen Beitrag zur Vermeidung unnötiger Gutachten oder gar Prozesse zu leisten.

\section{Fehlervermutungen durch nachbehandelnde Ärztinnen}

Viele Patientinnen wurden erstmals von nachbehandelnden Ärztinnen auf die Frage hingewiesen, ob es nicht angezeigt wäre, eine frühere Behandlung begutachten zu lassen. Oft erweisen sich diese Hinweise nach Vorliegen des Gutachtens als zutreffend.

Hingegen fällt auf, dass eine operative Klinik in der deutschen Schweiz, ein Chefarzt einer anderen Deutschschweizer Klinik sowie einige wenige praktizierende Ärztinnen in verschiedenen Landesteilen Patientinnen recht zahlreich einer Anwältin oder der Gutachterstelle zuweisen - wobei die Gutachterinnen dann einigermassen oft doch keinen Fehler feststellen. Resultat dieser lockeren «Indikationsstellung» zum Gutachten: Pro Fall lösen sich für die Patientin 3000 bis 4000 Franken Anwältinnenkosten in Luft auf. Und die Gutachterin oder das Gutachterteam setzen zwanzig bis vierzig Stunden Arbeit in den Sand. 
Wir möchten den so grosszügig zur Haftpflichtabklärung zuweisenden Stellen vorschlagen, sicherzustellen, dass die Patientinnen sie in der Folge über das Ergebnis unterrichten. Damit können sie die Erfolgsrate ihrer Empfehlungen prospektiv überwachen und gegebenenfalls Konsequenzen ziehen. Denn eines ist klar: Wenn in einer nachoperierenden Klinik der Patientin mitgeteilt wird, man habe laufend die Fehler der andern Spitäler oder Ärztinnen auszubügeln, dann kann auch die erfahrene und sorgfältige Patientenanwältin ein aus ihrer Sicht nicht indiziertes Gutachten nicht mehr verhindern - die Patientin wird sie fragen, ob sie es denn wirklich besser wissen könne als die nachbehandelnde Klinik.

\section{Arbeit an neuem Reglement; Einsetzung wissenschaftlicher Beirat}

Im Sommer 2001 wurde der Entwurf für ein revidiertes Reglement in die interne und externe Vernehmlassung (Patientenorganisationen, Anwältinnen, Versicherern etc.) gegeben. Parallel wurden alle internen Abläufe kritisch hinterfragt. Im August hat der FMH-Zentralvorstand einen wissenschaftlichen Beirat eingesetzt, der bereits zur Analyse der Vernehmlassungsergebnisse Stellung nehmen konnte, bevor der definitive Reglementvorschlag und die Skizze der neugeordneten internen Abläufe im November 2001 in den Zentralvorstand gegeben wurden.

Die Inkraftsetzung des revidierten Reglements erfolgte im Februar 2002 mit der Publikation in der Schweizerischen Ärztezeitung [5].

Wir hoffen, dass die revidierten Bestimmun-

5 Zur Revision Reglement FMHGutachterstelle Schweiz Ärztezeitung 2002;83(4):125-33. Die Unterlagen zur Gutachterstelle sind ebenfalls mit vielen ergänzenden Hinweisen auf der FMHWebsite zu finden: www.fmh.ch; Gutachterstelle.

6 Adresse pour la Romandie: Mme Brigitte Mottet, responsable du Bureau d'expertises extrajudiciaires de la FMH, case postale 64, 1010 Lausanne; tél. 0216521674 ; fax 0216523385 . gen in Zukunft zu einer klareren Ausgangslage führen, was sowohl die Qualität wie das Tempo des Begutachtungsverfahrens verbessern wird. Erste - nicht mehr in die Berichtsperiode fallende - Erfahrungen sind positiv.

\section{Dank}

Ich danke den Gutachterinnen, insbesondere aber auch den Delegierten Ärztinnen der Fachgesellschaften und den beiden Leiterinnen der Gutachterstelle Bern und Lausanne, für ihre grosse Arbeit im Interesse aller beteiligten Parteien.
Dass überall, wo gearbeitet wird, Fehler unterlaufen können, ist eine banale Erkenntnis. Dieser Erkenntnis nachzuleben ist jedoch für die direkt Betroffenen durchaus nicht banal. Ein aufrichtiger Dank geht deshalb ebenfalls an die vielen Ärztinnen und Spitäler, die bei der von der Patientin beantragten gutachterlichen Abklärung offen und fair mitwirken.

\section{Telefonische Vorbesprechung, Adresse, Unterlagen}

Die FMH-Gutachterstelle ermöglicht seit Jahren den Patientinnen, ihren Anwältinnen und auch den anderen Personen, die die Patientin beraten, den Fall vor Einreichung des definitiven Gutachterantrags telefonisch mit der Leiterin der Gutachterstelle zu besprechen: Wo und bei wem erscheint aufgrund der bisherigen Vorabklärungen ein Fehler plausibel? An welche weiteren potentiellen Fehlerquellen sollte noch gedacht werden? Worin könnte der Gesundheitsschaden bestehen? Auf welche besonderen Aspekte soll die Gutachterstelle die Delegierte der Fachgesellschaft hinweisen, die einen Gutachtervorschlag unterbreiten muss? usw. Diese Vorbesprechungen benötigen vielleicht eine halbe oder eine ganze Stunde Zeit - damit kann aber wertvolle Zeit im Gutachterverfahren gewonnen und können viele Rückfragen vermieden werden.

Die Unterlagen für die Einreichung eines Antrags auf Begutachtung sind für die deutsche Schweiz und das Tessin [6] erhältlich bei: Frau Susanne Friedli, Leiterin der FMH-Gutachterstelle, Postfach 293, 3000 Bern 16, Tel. 03131208 77, Fax 0313119981.

Die vollständigen Unterlagen können zurzeit auch auf der Website der FMH eingesehen werden: www.fmh.ch; Gutachterstelle. (Hinweis: Wir hoffen, dass die Zugänglichkeit der Unterlagen auf der Website nicht dazu führt, dass die wichtige telefonische Vorbesprechung des Falls mit der Leiterin der Gutachterstelle unterlassen wird; sonst müssten wir wieder zum System des ausschliesslich schriftlichen Versands der Unterlagen zurückkehren.) 